searchers reported. At temperatures of $400^{\circ} \mathrm{C}$ and lower, the spectra show the formation of cationic gold, which is active for the oxidation of hydrogen in the water gasshift reaction $\left(\mathrm{CO}_{2}+\mathrm{H}_{2}=\mathrm{CO}+\mathrm{H}_{2} \mathrm{O}\right)$.

The investigators concluded that the optimal process for obtaining the selective catalyst must result in the formation of metallic gold in $\mathrm{Fe}_{2} \mathrm{O}_{3}$ particles and they established a two-stage process: $3 \mathrm{~h}$ at $400^{\circ} \mathrm{C}$ and $2 \mathrm{~h}$ at $550^{\circ} \mathrm{C}$. The researchers demonstrated a heterogeneous catalyst that remained stable for more than $80 \mathrm{~h}$ of continuous use. This development, said the research team, has the potential benefit of eliminating the need for a multistage reactor currently required to eliminate $\mathrm{CO}$ from the reformer fuel.

SIARI SOSA

\section{Ultrafast Laser-Driven \\ Microfocus X-Ray Plasma Source Increases Time Resolution in Diffraction Experiments}

Ultrafast laser technology involves the use of a femtosecond laser, with the potential of producing laser pulses with enormous peak powers and power densities. High peak-power ultrafast laser pulses have been used at a number of facilities to generate ultrafast $\mathrm{x}$-ray pulses from laserinduced plasmas. Such technology facilitates the study of very fast chemical reactions and their intermediate and transition states. When an ultrafast laser with a small spot size is applied in a diffraction experiment, the temporal resolution of the response depends on the ability to control the jitter between the x-ray pulse and the laser pulse. This is the idea behind a broadband microfocus x-ray source designed as a result of the collaboration between a research group from the Max Born Institute led by T. Elsaesser and another from the Friedrich Schiller University led by E. Förster as described in the July 1 issue of Optics Letters (p. 1737).

The experimental setup for the microfocus $x$-ray source included a $1 \mathrm{kHz}$ Ti:sapphire laser source with 45 fs duration laser pulses incident upon a $20-\mu \mathrm{m}$ thick copper foil, which was spooled such that it could be moved with precision so that each laser pulse was incident upon a fresh surface. When the laser intensity exceeded $10^{12} \mathrm{~W} / \mathrm{cm}^{2}$, a high-temperature plasma developed at the target. At intensities higher than $10^{16} \mathrm{~W} / \mathrm{cm}^{2}$, hot electrons were created that penetrated the target, generating incoherent $\mathrm{x}$-rays. Similar levels of $\mathrm{Cu} \mathrm{K} \mathrm{K}_{\alpha}$ flux were detected by the investigators in both transmitted and reflected directions, increasing with laser intensity to a maximum of $6.8 \times 10^{10}$ photons $/ \mathrm{s}$, remaining stable in a $10 \mathrm{~h}$ span. The transmission geometry has two main advantages over the standard reflection geome- try, according to N. Zhavoronkov of Max Born Institute, "The main advantages of this new setup are the possibility to determine the initial pulse time point with very good accuracy, and a significantly diminished temporal jitter, because of the novel transmission geometry introduced."

An image of the x-ray emitting area captured with a CCD and measured using a knife-edge technique gave a source diameter after deconvolution of $10 \mu \mathrm{m} \pm 2 \mu \mathrm{m}$ full width at half maximum (FWHM). A more direct measurement using a toroidal $\mathrm{Ge}(444)$ single crystal as an x-ray focusing mirror gave a source diameter of $23 \mu \mathrm{m}$ $\pm 13 \mu \mathrm{m}$. Such small size and accuracy for an $\mathrm{x}$-ray source allows the performance of diffraction experiments with improved resolution over that obtained with traditional x-ray sources.

Using this setup to measure the response of a semiconductor placed in the transmitted beam, the duration of the x-ray pulse was estimated at no more than 500 fs from the resulting cross-correlation of the pump (laser) and probe (x-ray) pulses. This represents an improvement on time resolution for diffraction experiments, with a demonstrated increase in the $\mathrm{Cu}$ $\mathrm{K}_{\alpha}$ flux. Such an advance has potential not only for x-ray microscopy, but also for phase-contrast radiography and clinical applications such as microtomography.

SIARI SOSA

\section{Opal Strips Fabricated by Capillary-Assisted Deposition}

Colloidal photonic crystals have recently attracted attention because of their ability to control light propagation. A popular method for preparing these synthetic opal photonic crystals is the self-assembly of colloidal microspheres. For advanced applications of colloidal crystals in light-controlling devices, there is a need to introduce elements of optical circuit architectures within them. As reported in the July 26 issue of Chemistry of Materials (p. 3809; DOI: 10.1021/ cm051114i), H.-L. Li and F. Marlow of the Max-Planck-Institut für Kohlenforschung in Mülheim an der Ruhr, Germany have utilized capillary forces to fabricate alternating strips of opal from a colloidal suspension of polystyrene microspheres.

The opal strips were grown in a planar capillary cell, which was constructed by separating two parallel glass slides by a thin spacer. The bottom glass slide had a $1 \mathrm{~mm}$ hole, and the polystyrene microspheres were transferred to the cell by connecting this hole to a capillary tube immersed in the colloidal solution. Capillary forces assisted in delivering the

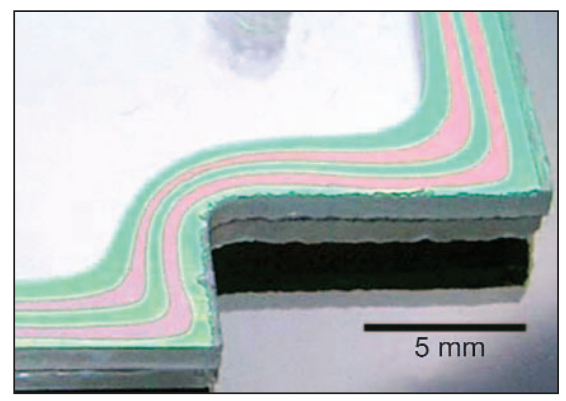

Figure 1. Alternating strip opal heterostructures obtained inside capillary cells with a special shape. Reprinted in part with permission from Chemistry of Materials 17(15)(2005) p. 3809; DOI: 10.1021/cm051114i. (c) 2005 American Chemical Society. suspension to the horizontal cell; the opal strips were deposited along the edges of the bottom glass slide upon solvent evaporation. By alternately dipping the capillary tube in two different solutions, the researchers were able to create up to 20 strips of opal comprising $287 \mathrm{~nm}$ and 345 $\mathrm{nm}$ polystyrene spheres. The thickness of these strips can be tuned by changing the spacer thickness, while their widths can be controlled by adjusting the polystyrene concentration and the infiltration time.

These opal heterostructures exhibit multiple reflection peaks which correspond to the stop bands of the constituent opals comprising the strips. It is possible to shape the opal strips by using a non-rectangular capillary cell since the growth starts along the edge of the glass slide (see Figure 1). Marlow said that this is one of the first attempts to create bent opal strips without the use of a complicated template.

TUSHAR PRASAD 